\title{
Une méthode stochastique pour la prédétermination des fluctuations probables des durées de service des réservoirs collinaires en Tunisie
}

\section{A stochastic method for the predetermination of the probable fluctuations in service duration of hill reservoirs in Tunisia}

\author{
Y. Zahar et J. P. Laborde
}

Volume 11, numéro 1, 1998

URI : https://id.erudit.org/iderudit/705295ar

DOI : https://doi.org/10.7202/705295ar

Aller au sommaire du numéro

Éditeur(s)

Université du Québec - INRS-Eau, Terre et Environnement (INRS-ETE)

ISSN

0992-7158 (imprimé)

1718-8598 (numérique)

Découvrir la revue

Citer cet article

Zahar, Y. \& Laborde, J. P. (1998). Une méthode stochastique pour la prédétermination des fluctuations probables des durées de service des réservoirs collinaires en Tunisie. Revue des sciences de l'eau / Journal of Water Science, 11(1), 25-42. https://doi.org/10.7202/705295ar

\section{Résumé de l'article}

Un modèle de génération stochastique de pluies est couplé ^ un modèle de calcul de l'index de leurs érosivités, dérivé de l'Equation Universelle de l'Erosion des Sols (USLE). Le premier fonctionne au pas de temps de 30mn, il est calé sur une série pluviographique de 15 ans de la Tunisie centrale. Le second modèle fonctionne par calcul automatique des cumuls et moyennes de l'érosivité des pluies générées.

En mode opérationnel, ces deux modèles sont exploités pour simuler les aléas de l'envasement annuel des réservoirs collinaires de la zone aride et semi-aride de la Tunisie : le bassin versant est considéré comme une "boite noire" où l'agressivité climatique est la principale variable (quelques pluies extrêmes font l'essentiel de l'érosion), les autres facteurs sont considérés constants durant la durée de service du réservoir.

Nous observons sur trois bassins versants répartis du nord au sud de la frange comprise entre $500 \mathrm{~mm}$ et $250 \mathrm{~mm}$ (de pluie moyenne annuelle), que la distribution annuelle des index d'érosivité des pluies peut être assimilée ^ la distribution des transports solides.

Sur l'un de ces bassins versants (OUED EL HISSIANE : 15,9) nous observons également que les valeurs extrêmes de l'érosion sont proportionnelles aux valeurs extrêmes de l'index d'érosivité des pluies. Seulement l'automne et le printemps sont des saisons érosives.

Dans le cas de petits bassins versants non-jaugés, comme ceux pour l'aménagement de réservoirs collinaires, le générateur nous permet de constituer des chroniques d'érosivité de pluie. Si on considère que les autres paramètres sont constants, ce modèle nous aide à déterminer les intervalles de confiance de durées de service probables.

Une analyse de sensibilité par la modification des paramètres du générateur (nombre d'épisodes, hauteur de pluie, maximum et durée d'averse etc ...) valide la méthodologie. De même une analyse régionale montre les faibles fluctuations des résultats sur l'étendue aride et semi-aride de la Tunisie.

Ces deux résultats nous ont conduit à proposer un abaque régional de prédétermination des fluctuations probables des durées de service des réservoirs collinaires, compte tenu de la connaissance préalable de la durée de service moyenne probable.

Cette méthode directement opérationnelle peut être utilisée pour l'aménagement, la planification, et la gestion des réservoirs collinaires. Elle améliore les études de faisabilité, notamment lorsqu'on la couple aux calculs économiques. 


\title{
Une méthode stochastique pour la détermination des fluctuations probables des durées de service des réservoirs collinaires en Tunisie
}

\author{
A stochastic method for the predetermination \\ of the probable fluctuations in service duration \\ of hill reservoirs in Tunisia
}

Y. ZAHAR ${ }^{1}$ et J.P. LABORDE 2

Reçu le 25 mars 1996, accepté le 7 octobre 1997*.

\section{SUMMARY}

A stochastic rainfall generator and a model for calculating the erosivity index of the Universal Soil Loss Equation (USLE) are coupled. The first one operates on a 30 min time step and it is calibrated on a 15 year historical pluviographic database for central Tunisia. The second model works like an automatic calculator, where the rain erosivity index can be averaged or summed.

These two models are applied to simulate the annual siltation of hill reservoirs in arid and semi-arid areas of Tunisia. The catchment is considered as a "black-box" where mostly climatic aggressivity is varying (some extreme rainfall events cause most of the erosion) and the other erosion parameters are considered to remain constant over the lifetime of the reservoir.

We know that the Wischmeier rain erosion index has no upper limit. When all the other factors are maintained constant, erosion is proportional to it. This climatic index is calculated for each rain event, but we can also calculate the annual average or sum it over many years. Soil losses (or solid transport) during a period are proportional to the value of this index summed over the same period.

We demonstrate on three representative catchments lying from north to south (from $500 \mathrm{~mm}$ to $250 \mathrm{~mm}$ annual average rainfall) that the annual rain erosion index distribution can be considered comparable to the solid transport distribution (Galton distributions with equal statistical parameters).

For the oued EI Hissiane catchment $\left(15.9 \mathrm{~km}^{2}\right)$, the annual extreme value of erosion is proportional to the annual extreme value of the rain erosion index. Only spring and autumn storms need be considered to calculate the annual sediment loss.

1. Université de Tunis 1 . Faculté de La Manouba, 2010 La Manouba, Tunis, Tunisie.

2. Université de Nice Sophia-Antipolis. Laboratoire d'Analyse Spatiale, 98 Bd Édouard Herriot, BP 209, 06204 Nice Cedex 3, France.

* Les commentaires seront reçus jusqu'au 6 novembre 1998. 
On gauged small catchments like those of hill reservoirs, the rainfall generator can provide an erosivity rainfall record. Considering that the other erosion parameters are constant, this model allows one to calculate the confidence intervals of a reservoir's predicted durations. We equate the annual erosivity index distribution to the annual solid supplies distribution, and we generate and sum over several consecutive years many series of "solid supplies" to simulate the progressive silting up of the reservoirs (represented by the chosen period: sum over $T=5$ years, 10 years, 15 years...). In the context of setting up hill reservoirs, this study concerns the potential duration and possible services of hydraulic structures (including the risk of rapid silting up of the reservoirs). This generator can be computed over many years and avoids the limitation due to the short observed time series.

The methodological benefit of such an approach is therefore to avoid the difficulty of limited observed samples. It enlarges the data analysis of the series dispersal and residuals. This approach, based on observations and a statistical method, supports experimental analysis. It gives a clear idea of the rate of siltation, according to climatic hazards.

A sensitivity analysis, where we modify the rainfall generator parameters (number of episodes and quantity of rain, peak and duration of storm...), is used to validate the methodology. A regional analysis shows a very low fluctuation considering the whole arid and semi-arid area of Tunisia. These two results led us to set up a regional abacus. This abacus reports the forecasts of the fluctuations of the time length service for hilly and small dams, including the probable average of their service duration. It allow a predetermination of the small dams' life time together with a confidence interval, and it can be used for small dam construction, planning and management. It improves the first feasibility study and can be included in economic calculations.

Key-words : Tunisia, arid and semi-arid climate, stochastic generation, erosion, Wischmeïer index, hill reservoirs, service duration.

Un modèle de génération stochastique de pluies est couplé à un modèle de calcul de l'index de leurs érosivités, dérivé de l'Équation Universelle de l'Érosion des Sols (USLE). Le premier fonctionne au pas de temps de $30 \mathrm{mn}$, il est calé sur une série pluviographique de 15 ans de la Tunisie centrale. Le second modèle fonctionne par calcul automatique des cumuls et moyennes de l'érosivité des pluies générées.

En mode opérationnel, ces deux modèles sont exploités pour simuler les aléas de l'envasement annuel des réservoirs collinaires de la zone aride et semi-aride de la Tunisie : le bassin versant est considéré comme une * boîte noire * où l'agressivité climatique est la principale variable (quelques pluies extrêmes font l'essentiel de l'érosion), les autres facteurs sont considérés constants durant la durée de service du réservoir.

Nous observons sur trois bassins versants répartis du nord au sud de la frange comprise entre $500 \mathrm{~mm}$ et $250 \mathrm{~mm}$ (de pluie moyenne annuelle), que la distribution annuelle des index d'érosivité des pluies peut être assimilée à la distribution des transports solides.

Sur l'un de ces bassins versants (oued El Hissiane : $15,9 \mathbf{~ k m}^{2}$ ) nous observons également que les valeurs extrêmes de l'érosion sont proportionnelles aux valeurs extrêmes de l'index d'érosivité des pluies. Seulement l'automne et le printemps sont des saisons érosives. 
Dans le cas de petits bassins versants non-jaugés, comme ceux pour l'aménagement de réservoirs collinaires, le générateur nous permet de constituer des chroniques d'érosivité de pluie. Si on considère que les autres paramètres sont constants, ce modèle nous aide à déterminer les intervalles de confiance de durées de service probables.

Une analyse de sensibilité par la modification des paramètres du générateur (nombre d'épisodes, hauteur de pluie, maximum et durée d'averse etc.) valide la méthodologie. De même une analyse régionale montre les faibles fluctuations des résultats sur l'étendue aride et semi-aride de la Tunisie.

Ces deux résultats nous ont conduit à proposer un abaque régional de prédétermination des fluctuations probables des durées de service des réservoirs collinaires, compte tenu de la connaissance préalable de la durée de service moyenne probable.

Cette méthode directement opérationnelle peut être utilisée pour l'aménagement, la planification, et la gestion des réservoirs collinaires. Elle améliore les études de faisabilité, notamment lorsqu'on la couple aux calculs économiques.

Mots clés : Tunisie, climat aride et semi-aride, génération stochastique, érosion, index de Wischmeïer, réservoirs collinatres, durées de senvice.

\section{1 - INTRODUCTION}

Les phénomènes d'érosion, de transport et de sédimentation des ouvrages de mobilisation des eaux de surface, sont très importants dans l'aménagement hydraulique en Tunisie, mais sont encore assez insuffisamment connus à l'échelle des petits bassins versants, généralement non-jaugés. Nous nous sommes proposés de contourner le manque de données opérationnelles de terrain (plusieurs chroniques de débits solides statistiquement exploitables), par la génération stochastique d'épisodes pluvieux sur de longues périodes, ainsi que de leur érosivité, calculée à partir de l'index « $R$ » de WISCHMEIER (1959). Nous savons que, par définition, cet index d'érosivité des pluies se cumule dans le temps tout comme l'érosion mesurable, et que si tous les autres facteurs de l'érosion sont maintenus constants (à l'exception de la pluie) le cumul de l'érosion sur une période donnée, est proportionnel au cumul de R (MASSON, 1980). Ainsi, ayant assimilé la distribution statistique des séries annuelles d'index d'érosivité à celle des séries annuelles d'apports solides (mêmes fonctions de répartition, et paramètres statistiques sensiblement égaux), on a pu générer plusieurs échantillons indépendants de différentes tailles $(5,10,15$ ans...), et cumuler l'index de l'érosion sur chacune de ces périodes, représentant les capacités des réservoirs. La multiplication des échantillons pour chaque période, permet de comparer les fluctuations des cumuls d'une série à l'autre, et d'analyser ainsi leurs dispersions. Cette dispersion définie par des valeurs quantiliques, permet la détermination des fluctuations des durées de service probables, qui peuvent directement être utilisées dans les calculs de rentabilité. 


\section{2 - PROBLÉMATIQUE ET CADRE DE LA RECHERCHE}

Un important programme de mobilisation des eaux de surface est planifié en Tunisie pour la décennie (1991-2000): 21 barrages, 203 barrages collinaires, 1000 lacs collinaires, 4000 ouvrages d'épandage des eaux de crues. Ces ouvrages " de $2^{\mathrm{e}}$ génération " qui viennent après la réalisation des grands barrages, sont souvent à la limite de la faisabilité économique, et sont plus justifiés par l'intérêt social : développement rural et régional, amélioration des conditions de vie etc. (TALINEAU et al., 1994).

La grande majorité des réservoirs collinaires (lacs ou barrages collinaires selon la capacité de la retenue, généralement inférieure à respectivement 300000 et $1000000 \mathrm{~m}^{3}$ ) sont programmés en Tunisie aride et semi-aride, dans la frange comprise entre les isohyètes 250 et $500 \mathrm{~mm}$ (fig. 1). C'est là où les phénomènes d'érosion sont très fréquents, ils risquent par conséquent de connaître des durées de service relativement réduites. Actuellement, 26 lacs collinaires font l'objet d'un suivi annuel de leur envasement (depuis 1993). Le dernier annuaire hydrologique de ces ouvrages annonce le chiffre de 3,5\% pour la perte annuelle globale du volume mobilisable (ALBERGEL et REJEB, 1997). Mais, il faudra attendre quelques années encore avant que les chroniques d'envasement progressif soient suffisamment exhaustives (10 à 15 années de service au moins).

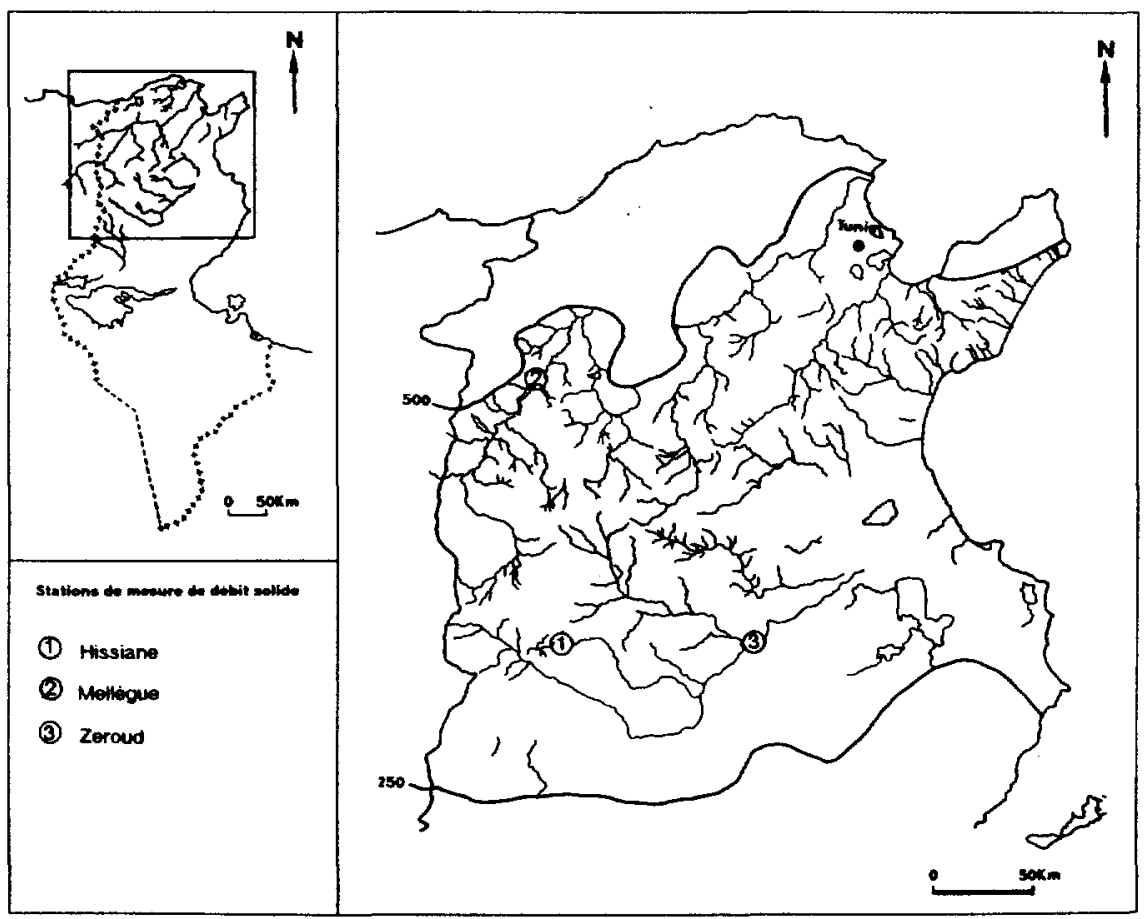

Figure 1 Zone semi-aride et aride d'implantation des lacs et barrages collinaires.

The location of hill dams in the arid and semi-arid areas. 
À la demande d'une évaluation de la durée de service d'un ouvrage apparaît celle de fixer l'intervalle quantilique dans lequel se situerait cette durée de service préalablement estimée. Pour cela, on fait l'hypothèse que cet intervalle dépend essentiellement de l'occurrence des événements pluvieux extrêmes.

\section{3 - DONNÉES ET MÉTHODES}

\subsection{Hypothèses et validation}

Trois hypothèses sont préalables à nos travaux. Elles s'appuient sur des observations souvent admises par des recherches antérieures ou en cours (HEUSCH, 1970 ; E. ROOSE, 1976 ; VOGT, 1991).

\section{Hypothèse 1 - Les saisons érosives sont l'automne et le printemps.}

Le dépouillement de 11 années (1978-1988) de mesures de transport solide en suspension sur oued El Hissiane $\left(15,9 \mathrm{~km}^{2}\right)$ dans le Djebel Semmama en Tunisie centrale (POUYAUD et al., 1995), a fait ressortir que les mois de septembre et de juin sont les plus érosifs. Ils dépassent assez significativement les autres mois (fig. 2).

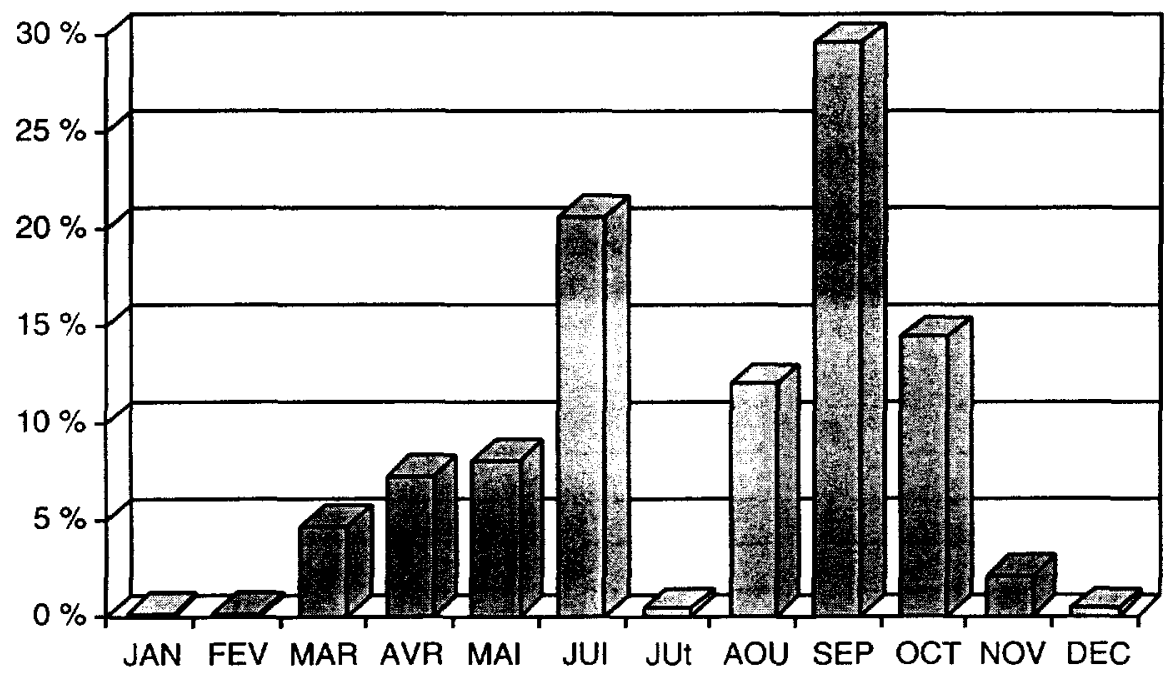

Figure 2 Pourcentages mensuels du transport solide en suspension.

Monthly percentage of sediment transport.

Si nous classons le mois de juin avec le reste des mois du printemps, et le mois d'août avec les mois d'automne (le mois d'août est érosif par quelques pluies orageuses particulièrement violentes dans sa dernière décade), on obtient deux saisons érosives de 4 mois chacune :

- L'automne (d'août à novembre) avec $54 \%$ du transport solide en suspension.

- Le printemps (de mars à juin) avec $45 \%$. 
Le mois de juillet et les trois mois de l'hiver, semblent ne pas constituer de risques véritables (à peine $1 \%$ des mesures), nous pouvons les négliger.

Hypothèse 2 - Les pluies extrêmes sont la cause principale de l'érosion, et à l'échelle des petits bassins versants (inférieurs ou de l'ordre de $100 \mathrm{~km}^{2}$ ), l'index d'érosivité des pluies est encore assez étroitement liée à l'érosion mesurable qui lui est proportionnelle.

Certes, d'une façon générale les phénomènes sont beaucoup trop complexes, entre pluie-transport-érosion et sédimentation, et entre les diverses caractéristiques des pluies (hauteur, durée, intensités maximales, saisons etc.), et l'érosion mesurable (MORGAN, 1986). Mais en zone aride et semi-aride par contraste avec la rareté des précipitations, il a souvent été observé que quelques pluies extrêmes font l'essentiel de l'érosion, on cite notamment :

DELHOUME (1985) « 3,5\% des pluies totales mesurées entre 1976 et 1981 provoquent 50 à $75 \%$ de l'érosion totale mesurée " sur parcelles expérimentales en Tunisie centrale.

BOURGES et BOUZAIANE (1973) Une averse a provoqué $44 \%$ de l'érosion totale obtenue en 5 ans d'observation sur un bassin versant du sud tunisien de $2,2 \mathrm{~km}^{2}$ ('’oued Zita).

Si on considère les pluies maximales annuelles, comme des pluies extrêmes (événements indépendants et saisonniers), on peut sélectionner à la fois ces événements, et les valeurs de transport solide en suspension correspondantes. On observe, pour oued El Hissiane, que la majorité de ces pluies engendrent “ l'événement érosif maximal de l'année" (9 sur 11 plus exactement). Par ailleurs, ces quelques événements érosifs extrêmes totalisent en effet souvent, un fort pourcentage de l'apport total annuel en sédiment (fig. 3).

À eux seuls, ces 11 événements ont contribué à $59 \%$ de l'érosion totale mesurée (les 11 plus fortes valeurs de la série ne totalisent pas plus que $66 \%$, mais 6 d'entre elles ont fait $50 \%$ du cumul de 11 ans). La dynamique érosive semble donc régie principalement par les pluies extrêmes, ou maximales annuelles, qui

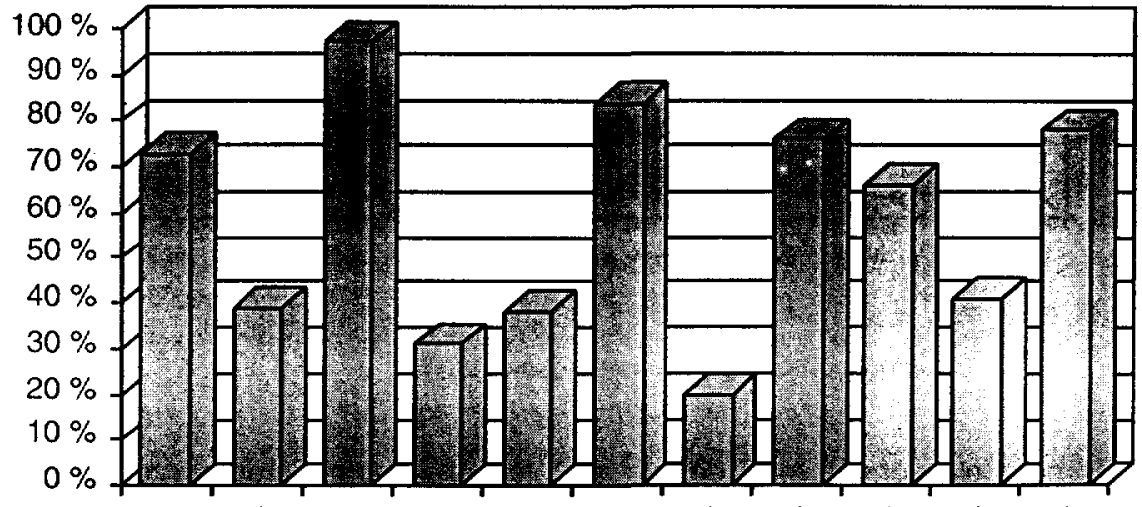

$77 / 78$ 78/79 79/80 80/81 81/82 82/83 83/84 84/85 85/86 86/87 87/88

Figure 3 Contribution de l'événement maximum annuel dans l'apport total annuel de sédiments (\%).

Contribution of the maximal annual event to the total annual sediment supply. 
sont d'ailleurs assez récurrentes d'une année à l'autre, mais plus fréquentes à l'automne qu'au printemps. Ainsi, on admet et on vérifie, qu'à l'occasion de ces pluies extrêmes, autant les index d'érosivité que les érosions mesurées correspondantes, obtiennent des valeurs records (ZAHAR, 1994).

Une corrélation entre la moyenne spatiale des index d'érosivité annuels calculés aux postes pluviographiques, et l'érosion annuelle mesurée à l'aval de l'oued El Hissiane a été obtenue. La régression est pertinente visiblement par les quelques événements extrêmes (fig. 4).

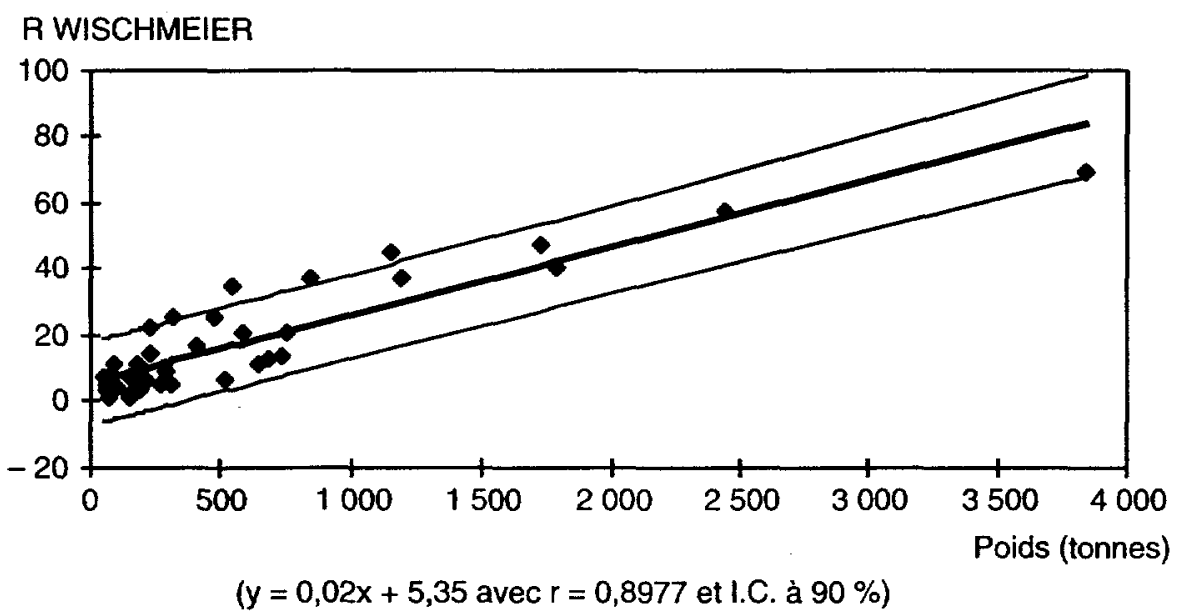

Figure 4 Régression linéaire entre transport solide en suspension et index $R$ des pluies.

Linear regression between sediment transport and the rainfall index $A$.

Nous avons choisi pour exprimer l'index d'érosivité de WISCHMEIER (1959), l'expression dans le système d'unité international, proposée par MASSON (1971):

$E$ : énergie cinétique en Joules $/ \mathrm{m}^{2}$.

$$
\mathrm{R}=\mathrm{K} \cdot \mathrm{E} \cdot \mathrm{I}_{30} \max
$$

$\mathrm{I}_{30}$ max : intensité maximale en 30 minutes pour une averse exprimée en $\mathrm{mm} / \mathrm{h}$.

$K$ : constante qui dépend du système d'unité $: K=1 / 685$ (système international).

Certes, la corrélation entre index d'érosivité des pluies et érosion mesurable, n'est valable en théorie qu'à l'échelle des parcelles expérimentales (WISCHMEIER et SMITH, 1958). Toutefois l'extrapolation devrait se limiter aux petits bassins versants de montagne de tailles inférieures ou de l'ordre de $100 \mathrm{~km}^{2}$ (ce qui est généralement le cas des bassins choisis pour l'aménagement des réservoirs collinaires). La faible taille des bassins versants conjuguée aux durées brèves des pluies orageuses et intenses d'automne ou de printemps, génèrent souvent des crues brutales et turbides.

Hypothèse 3 - Les distributions des cumuls annuels d'index d'érosivité ou de transport solide, ont des comportements statistiques voisins et de même ordre.

II n'est pas habituel de parler de fonction de répartition dans le cas d'études de débits solides. Probablement par manque de chroniques de taille suffisante pour 
tester un ajustement statistique. Ces chroniques sur de longues périodes, sont souvent difficilement réalisables, du fait même de la complexité et des erreurs des mesures et des prélèvements sur le terrain. À notre connaissance, à part la série de l'oued El Hissiane, deux autres séries de débits solides (chroniques annuelles de matières en suspension) peuvent être exploitées statistiquement :

- La série de l'oued Mellègue $\left(10308 \mathrm{~km}^{2}\right)$ qui correspond à la période (1954-1975) soit les 21 premières années de service du barrage (CLAUDE et CHARTIER, 1977).

- La série de l'oued Zeroud $\left(8950 \mathrm{~km}^{2}\right)$ qui correspond à la période qui précède la mise en eau du barrage Sidi Saâd, à des dates d'observation (19561978) à peu près concomitantes avec la série du Meliègue (BOUZAIANE et al., 1986).

Outre le fait qu'il s'agit de bassins versants de taille beaucoup plus grande, ces séries couvrent avec celle de l'oued El Hissiane, trois sites géographiques différents du nord au sud de la zone aride et semi-aride qui nous intéresse (fig. 1).

Les distributions de ces 3 séries étant nettement dissymétriques (dissymétrie positive engendrée par quelques valeurs maximales), elles semblent indiquer un comportement statistique à l'allure d'une loi log-normale (fig. 5, 6 et 7).

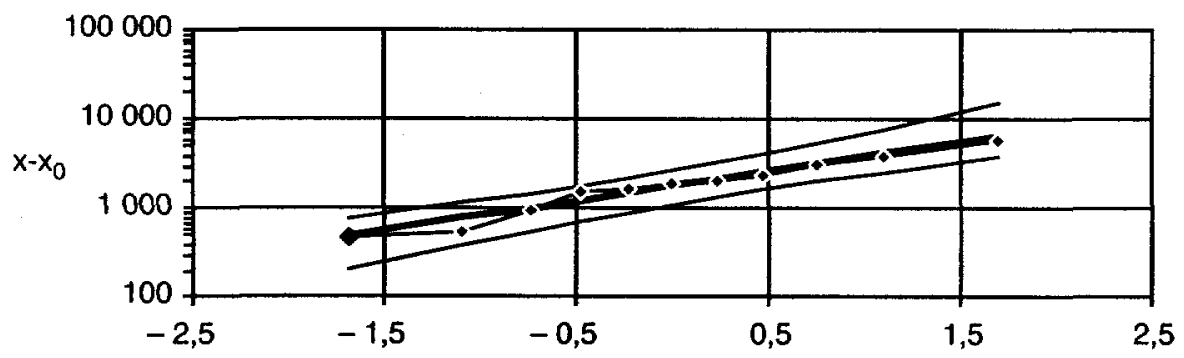

$\left(x_{0}=0,00\right.$ Moy. $\log \left(x-x_{0}\right)=3,2427$ E.T. $\log \left(x-x_{0}\right)=0,3330 n=11$ et I.C. à $\left.90 \%\right)$

Figure 5 Ajustement de la loi log-normale aux apports solides annuels d'El Hissiane.

A log-normal distribution fitted to the annual solid supply of the El Hissiane catchment.

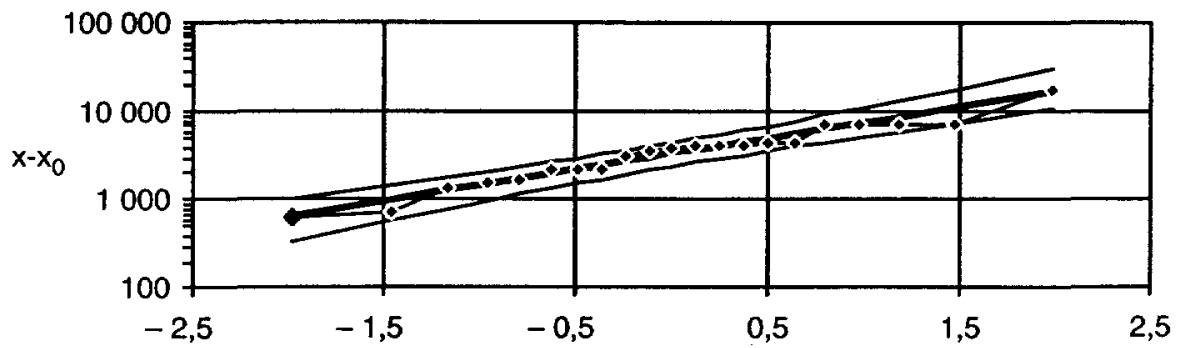

$\left(x_{0}=0,00\right.$ Moy. $\log \left(x-x_{0}\right)=3,5134$ E.T. $\log \left(x-x_{0}\right)=0,3524 n=21$ et I.C. à $\left.90 \%\right)$

Figure 6 Ajustement de la loi log-normale aux apports solides annuels du Mellègue.

A log-normal distribution fitted to the annual solid supply of the Mellègue catchment. 


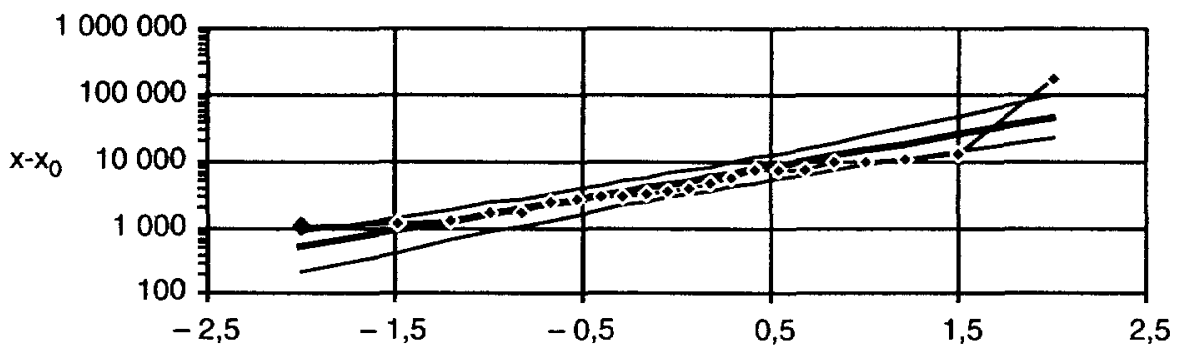

$\left(x_{0}=0,00\right.$ Moy. $\log \left(x-x_{0}\right)=3,6638$ E.T. $\log \left(x-x_{0}\right)=0,4814 n=22$ et I.C. à $\left.90 \%\right)$

Figure 7 Ajustement de la loi log-normale aux apports solides annuels du Zeroud.

A log-normal distribution fitted to the annual solid supply of the Zeroud catchment.

II apparaît toutefois qu'un point décroche dans le cas du Zeroud (fig. 7). Ce point est relatif aux crues très exceptionnelles du Zeroud en septembre-octobre 1969 (CRUETTE et RODIER, 1971). Cette valeur est en fait très approximative : les prélèvements effectués pendant les crues de 1969, l'ont été seulement sur les bords de l'oued, et les auteurs ont dû considérer que la turbidité moyenne correspondait à $10 \%$ du volume des crues. Nous avons par conséquent réajusté la loi log-normale à la série amputée de cette valeur (fig. 8).

On observe que la variabilité spatiale n'affecte pas, ou peu, le paramètre d'ajustement de la loi " $E$.T. $\log \left(x-x_{0}\right)$ ", qui est sensiblement constant dans les trois cas étudiés. À ce sujet, il a souvent été vérifié, lorsque le domaine d'étude est relativement peu vaste, et dans le cas de distributions log-normales de variables hydrologiques (pluies, débits etc.), que la variabilité spatiale n'affecte pas le paramètre a $E . T . \log \left(x-x_{0}\right)$ " (LABORDE, 1984).

Par ailleurs on a pu vérifier, malgré quelques cassures qui peuvent être affectées à la représentativité de la variable analysée par rapport à des données naturelles mesurées, ou à l'échantillonnage, que le comportement statistique des index d'érosivité cumulés annuellement à la station de l'oued El Hissiane, est en assez bon accord avec les distributions précédentes (fig. 9).

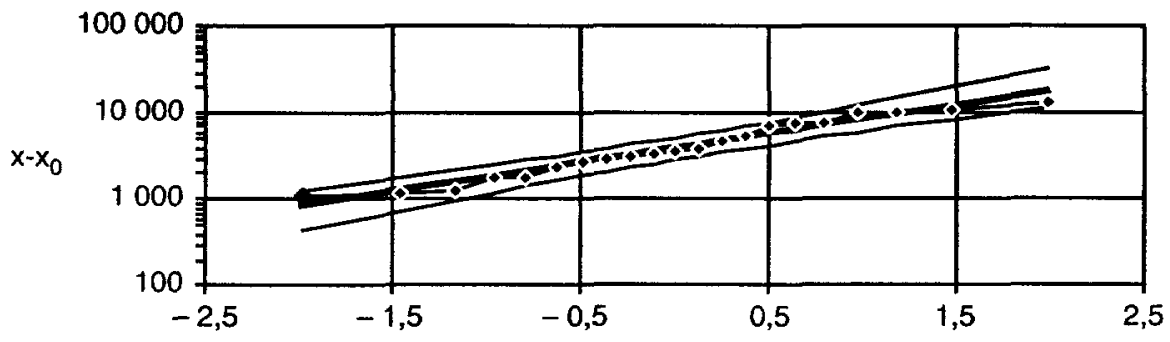

$\left(x_{0}=0,00\right.$ Moy. $\log \left(x-x_{0}\right)=3,5888$ E.T. $\log \left(x-x_{0}\right)=0,3367 n=21$ et I.C. à $\left.90 \%\right)$

Figure 8 Ajustement de la loi log-normale aux apports solides annuels du Zeroud (sans l'année 1969/70).

A log-normal distribution fitted to the annual solid supply of the Zeroud catchment (without 1969/70). 


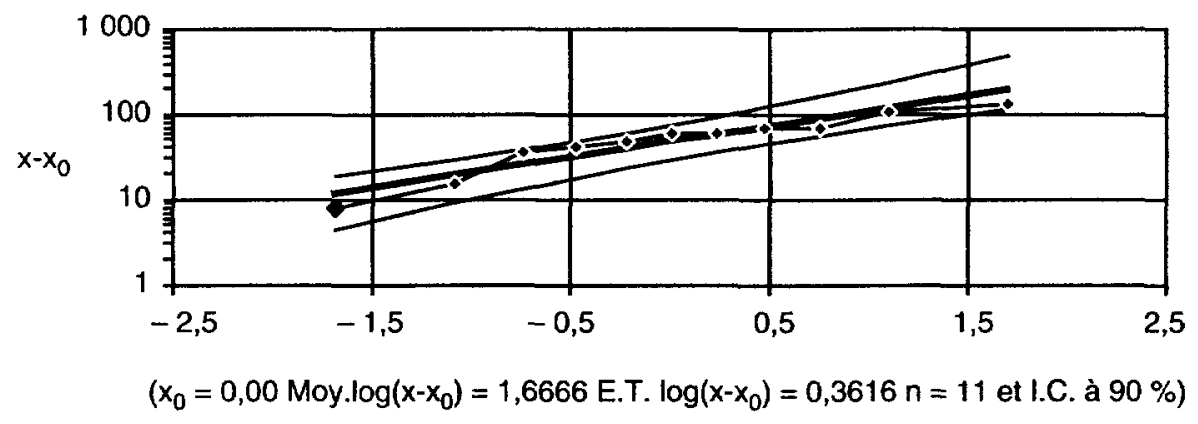

Figure 9 Ajustement de la loi-log normale aux index annuels d'érosivité d'El Hissiane.

A log-normal distribution fitted to the annual erosivity index of the El Hissiane catchment.

Le paramètre E.T.log $\left(x-x_{0}\right)$ reste à peu près constant. On obtient ainsi des valeurs récurrentielles proportionnelles avec celles des débits solides. En effet, si on compare chaque valeur récurrentielle à la valeur moyenne de l'échantillon, on observe que les rapports (en \%) sont sensiblement constants d'une série à l'autre, et encore dans le cas de la variable index d'érosivité (tabl. 1).

Tableau 1 Pourcentage de la valeur fréquentielle à la moyenne de l'échantillon observé.

Table 1 Percentage between the frequency value and the average of the observed sample.

\begin{tabular}{|lcccc|}
\hline Récurrences & $\begin{array}{c}\text { Érosivité R } \\
\text { El Hissiane }\end{array}$ & El Hissiane & Mellègue & Sidi Saâd \\
\hline $\mathrm{T}=2$ ans & $78 \%$ & $79 \%$ & $74 \%$ & $76 \%$ \\
$\mathrm{~T}=5$ ans & $158 \%$ & $150 \%$ & $147 \%$ & $147 \%$ \\
$\mathrm{~T}=10$ ans & $228 \%$ & $210 \%$ & $210 \%$ & $207 \%$ \\
$\mathrm{~T}=20$ ans & $308 \%$ & $277 \%$ & $282 \%$ & $274 \%$ \\
$\mathrm{~T}=50$ ans & $433 \%$ & $379 \%$ & $393 \%$ & $376 \%$ \\
$\mathrm{~T}=100$ ans & $543 \%$ & $467 \%$ & $490 \%$ & $464 \%$ \\
E.T. $\log \left(\mathrm{x}-\mathrm{x}_{0}\right)$ & 0,3616 & 0,3330 & 0,3524 & 0,3367 \\
\hline
\end{tabular}

La surestimation des valeurs pour l'index $\mathbf{R}$ par rapport à la série de transport solide à El Hissiane reste dans des proportions acceptables. On valide ainsi l'index d'érosivité des pluies d'une manière statistique, qui semble représenter assez fidèlement la dynamique érosive dans le temps.

On peut donc exploiter cette facilité de calcul dans un modèle de génération stochastique. Nous proposons une génération stochastique de la pluviographie qui sera traduite en index d'érosivité. À leur tour les index générés seront cumulés par saisons, par années et pour plusieurs années. Les aléas annuels de l'érosivité simulée, seront assimilés aux aléas de l'érosion mesurable, et serviront par déduction pour l'analyse des longévités probables des réservoirs, compte tenu surtout de l'occurrence des événements extrêmes. 


\subsection{Génération stochastique des pluies et de leurs érosivités}

La série pluviographique utilisée pour caler et valider le modèle de génération est celle du poste PG73 du bassin versant de l'oued El Hissiane, soit la plus longue, avec 15 années d'observation (1976-1990).

Pour des raisons d'homogénéité des pluies, nous proposons une génération stochastique par saison érosive ; l'automne et le printemps (les pluies de printemps sont notamment relativement plus durables que celles de l'automne). Pour reconstituer des statistiques annuelles nous pouvons à partir de la génération de deux séries saisonnières d'automne et de printemps de même taille, rassembler aléatoirement les valeurs saisonnières deux à deux pour en faire une série annuelle. En effet, nous avons observé que les pluies saisonnières d'automne et de printemps sont décorrélées $(r=0,22$, signification inférieure à $5 \%$ au sens du test de Student-Fisher).

Cette génération stochastique est obtenue à l'aide d'un modèle dérivé de celui mis au point par TOURASSE (1981), selon la schématisation des épisodes de pluie de TODOROVIC et YEVJEVICH (1969) : l'épisode pluvieux est découpé en une série d'averses, et le nombre d'averses "NA " en est une caractéristique. Le nombre d'épisodes NP, et le nombre d'averses NA sont tirées dans une loi de Poisson. Deux épisodes sont séparés par au moins 12 heures sèches, et chaque averse est séparée de la suivante par une durée sèche " $\mid A$ " d'au moins une heure trente minutes. Une averse est définie comme un ensemble d'intensités constantes générées par pas de temps de 30 minutes à l'aide des paramètres suivants :

- La durée d'averse DA (nombre entier multiple de 30 minutes).

- La hauteur de pluie de l'averse HA (en mm).

- Le rapport de l'intensité maximale en 30 minutes à l'intensité moyenne de l'averse RA (en \%).

- La position du maximum dans laverse PA (nombre entier multiple de 30 minutes). Chaque averse par hypothèse ne comporte qu'un seul maximum (c'est le cas pour $70 \%$ des averses observées).

Mis à part une liaison entre HA et DA (au printemps), qui nous a contraint à faire une distribution conditionnelle de $H A$, connaissant DA, les paramètres sont apparus indépendants les uns des autres et entre averses successives (ZAHAR, 1994). Les distributions expérimentales des paramètres énumérés ci-dessus ont été étudiés par saison, et des lois théoriques leur ont été ajustées (log-normale pour DA, HA et IA, normale pour RA).

La simulation se fait en utilisant un générateur de nombres aléatoires (tirage pseudo-aléatoire d'un nombre au hasard de la bibliothèque de l'ordinateur compris entre 0 et 1). La valeur de chaque paramètre est fixée par anamorphose entre la loi uniforme du générateur de nombres aléatoires et la loi théorique du paramètre concerné (fig. 10).

À chaque averse générée, un calcul automatique de l'érosivité s'en suit. Les résultats sont consignés en mémoire, pour être cumulés par épisodes et par saisons. La description détaillée de toute la chaîne de traitement est disponible dans la thèse de ZAHAR (1994).

La validation de cette génération a nécessité des variables auxiliaires qui n'interviennent pas dans le processus de simulation, telles que les hauteursdurées-fréquences, dont la superposition des courbes entre les données obser- 


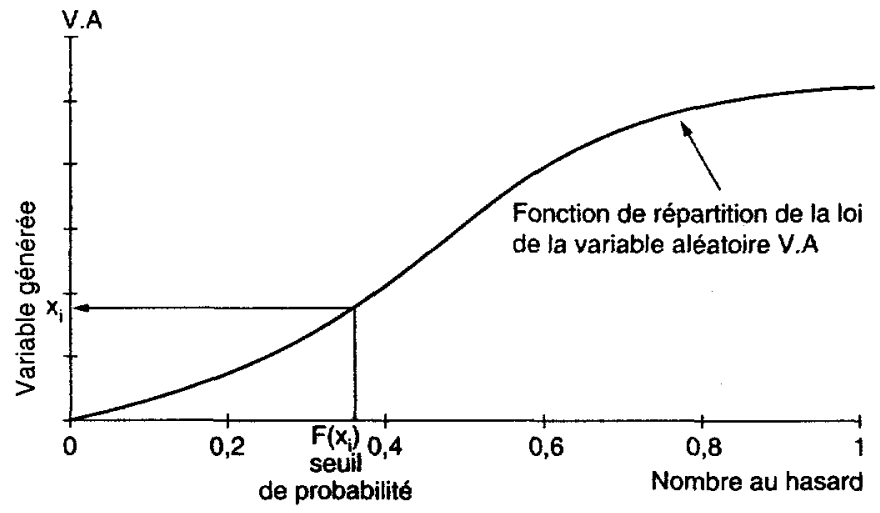

Figure 10 Principe de tirage aléatoire d'une variable.

Principle of a ramdom selection of a variable.

vées et générées par saison a montré de faibles écarts. Ci-joint la comparaison des valeurs décennales (tabl. 2 et 3 ).

De même on a observé que le modèle dans son ensemble reproduit assez fidèlement les caractéristiques de l'érosivité. En particulier, les distributions des cumuls annuels des index d'érosivité générés (agrégation des index saisonniers générés deux à deux) s'ajustent à la loi log-normale, et l'estimation des valeurs fréquentielles est reproduite relativement fidèlement par le générateur (tabl. 4).

Tableau 2 Comparaison des valeurs décennales observées et générées à l'automne.

Table 2 Comparison of decennial values observed and generated for the autumn.

\begin{tabular}{|lccccccccc|}
\hline Automne & $0,5 \mathrm{~h}$ & $1,5 \mathrm{~h}$ & $1 \mathrm{~h}$ & $2 \mathrm{~h}$ & $3 \mathrm{~h}$ & $6 \mathrm{~h}$ & $12 \mathrm{~h}$ & $18 \mathrm{~h}$ & $24 \mathrm{~h}$ \\
Observé & 20,8 & 22,3 & 24,3 & 26,3 & 27,7 & 31,4 & 35,5 & 36,5 & 40,0 \\
Généré & 22,8 & 23,8 & 24,9 & 25,6 & 27,7 & 29,4 & 30,9 & 32,4 & 33,4 \\
Écart (\%) & $+10 \%$ & $+7 \%$ & $+2 \%$ & $-3 \%$ & - & $-6 \%$ & $-13 \%$ & $-11 \%$ & $-16 \%$ \\
\hline
\end{tabular}

Tableau 3 Comparaison des valeurs décennales observées et générées au printemps.

Table 3 Comparison of decennial values observed and generated for the spring.

\begin{tabular}{|lccccccccc|}
\hline Printemps & $0,5 \mathrm{~h}$ & $1,5 \mathrm{~h}$ & $1 \mathrm{~h}$ & $2 \mathrm{~h}$ & $3 \mathrm{~h}$ & $6 \mathrm{~h}$ & $12 \mathrm{~h}$ & $18 \mathrm{~h}$ & $24 \mathrm{~h}$ \\
Observé & 18,4 & 21,9 & 25,2 & 26,9 & 29,4 & 37,1 & 51,3 & 52,3 & 59,7 \\
Généré & 19,4 & 25,0 & 27,3 & 29,3 & 31,6 & 35,9 & 42,2 & 43,9 & 45,2 \\
Écart $(\%)$ & $+5 \%$ & $+14 \%$ & $+8 \%$ & $+9 \%$ & $+7 \%$ & $-3 \%$ & $-18 \%$ & $-16 \%$ & $-24 \%$ \\
\hline
\end{tabular}

Tableau 4 Comparaison des statistiques des index annuels observés et générés.

Table 4 Comparison of the statistical annual index observed and generated.

\begin{tabular}{|lcccccc|}
\hline T (ans) & 2 & 5 & 10 & 20 & 50 & 100 \\
15 ans observés & 81 & 119 & 146 & 173 & 213 & 236 \\
100 ans générés & 73 & 113 & 142 & 172 & 218 & 246 \\
Écart $(\%)$ & $-10 \%$ & $-5 \%$ & $-3 \%$ & $-1 \%$ & $+2 \%$ & $+4 \%$ \\
\hline
\end{tabular}




\section{4 - RÉSULTATS}

On pose que la durée de service probable d'un réservoir est préalablement estimée à $n=10,20,50$ ans etc., et on génère aléatoirement des scénarios multiples de son envasement progressif, à l'aide des cumuls annuels d'index d'érosivité, assimilés donc à des apports de sédiments. En s'appuyant sur les hypothèses préalablement établies, et notamment sur le fait que les statistiques annuelles d'érosivité représentent assez fidèlement les statistiques de la variable transport solide, on peut simuler le comblement annuel du réservoir supposé existant, par la diminution progressive de sa capacité initiale qui est caractérisée par la valeur de " $n$ ». Cette simulation nous amène à l'analyse des aléas de durée de service de "louvrage ", en fonction de son comblement plus ou moins rapide, simplement par la multiplication des essais (fig. 11).

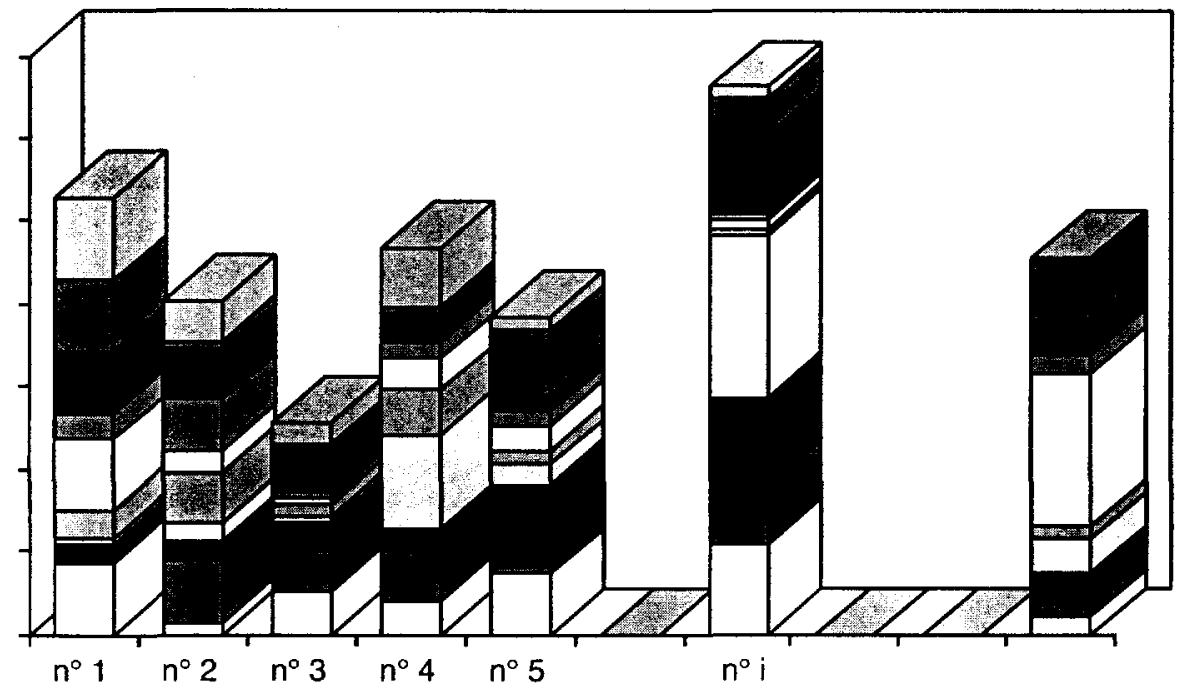

Figure 11 Index d'érosivité cumulé sur la période $\mathrm{n}$.

Erosivity index summed over a period $n$.

Pour chaque scénario “ $i$ " les apports annuels sont aléatoires, et plus ou moins importants, et d'un scénario à l'autre, les cumuls annuels sont également aléatoires et plus ou moins graves. On s'affranchit de cette manière des problèmes d'échantillonnage en générant à volonté des séries en nombre et en taille aussi grands que souhaitable, et on peut aborder de ce fait le problème sous l'aspect statistique.

Nous générons 1000 années, pour constituer des échantillons de cumuls de 5 ans, 10 ans... Pour chaque période de cumuls nous ajustons un modèle probabiliste (fig. 12). Le modèle qui s'ajuste le mieux est encore la loi log-normale.

Nous avons retenu différents seuils de probabilité, pour lesquels on calcule pour chaque période " $n$ ", le rapport de la valeur quantilique à la médiane. Nous 


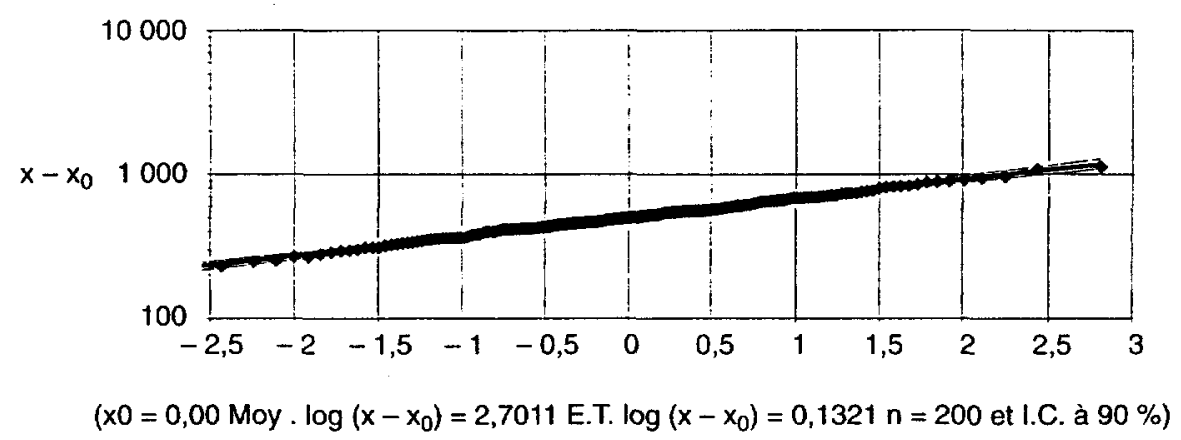

Figure 12 Ajustement à une loi log-normale des cumuls de l'index d'érosivité en 5 ans.

A log-normal distribution fitted to the results of the erosion index during a 5 year period.

avons baptisé ce rapport " coefficient de longévité ", pour désigner les écarts probables par rapport à la durée de service probable a priori estimée. II a été possible d'élaborer une formulation mathématique, à partir de la mise en application de ces résultats, et par l'ajustement aux coefficients de longévité isoquantiliques, de fonctions dont l'expression est de la forme :

$$
C_{l, F}=10^{\left(\alpha \cdot U_{F}\right)} /(\sqrt{n})
$$

$\mathrm{C}_{\mathrm{I}, \mathrm{F}}$ : coefficient de longévité.

$\mathrm{n}$ : durée de service probable.

$\mathrm{U}_{\mathrm{F}}$ : Variable de Gauss.

$\alpha$ : paramètre de calage régional, on trouve $\alpha=0,3$.

La valeur $\alpha=0,3$, est déduite par régression : en théorie les écart-types des séries sont, dans le cas de variables gaussiennes, proportionnelles à $1 / \sqrt{n}$. Nous avons par conséquent ajusté aux différents paramètres de la loi log-normale «E.T.log $\left(x-x_{0}\right)$ » qui correspondent, par analogie à une variable distribuée selon la loi de Gauss, à l'écart-type de la variable " normalisée " $\log \left(x-x_{0}\right)$, un modèle mathématique du type $1 / \sqrt{\mathrm{n}}$. Nous trouvons par cette régression la valeur $\alpha=0,3$, qui donne le faisceau de courbes de l'abaque suivant (fig. 13).

\subsection{Exemple d'application}

Supposons qu'un ingénieur calcule une durée de service moyenne probable d'un ouvrage $n=50$ ans. Ce calcul il l'a fait d'après le taux de comblement progressif observé après quelques années de service de l'ouvrage (10 ans et plus), ou à l'aide d'une formule empirique régionale, généralement en avant projet (GHORBEL, 1991). Il peut désormais analyser des scénarios de longévité probable, en faisant les calculs suivants (tabl. 5).

Ainsi, il peut affirmer que son ouvrage a $90 \%$ de chance pour que sa fin de service soit comprise dans l'intervalle 43 à 59 ans $(99 \%$ de chance de servir au moins 63 ans, ou $1 \%$ de chance de service moins de 40 ans). Enfin, il peut choisir tout autre intervalle de confiance. 


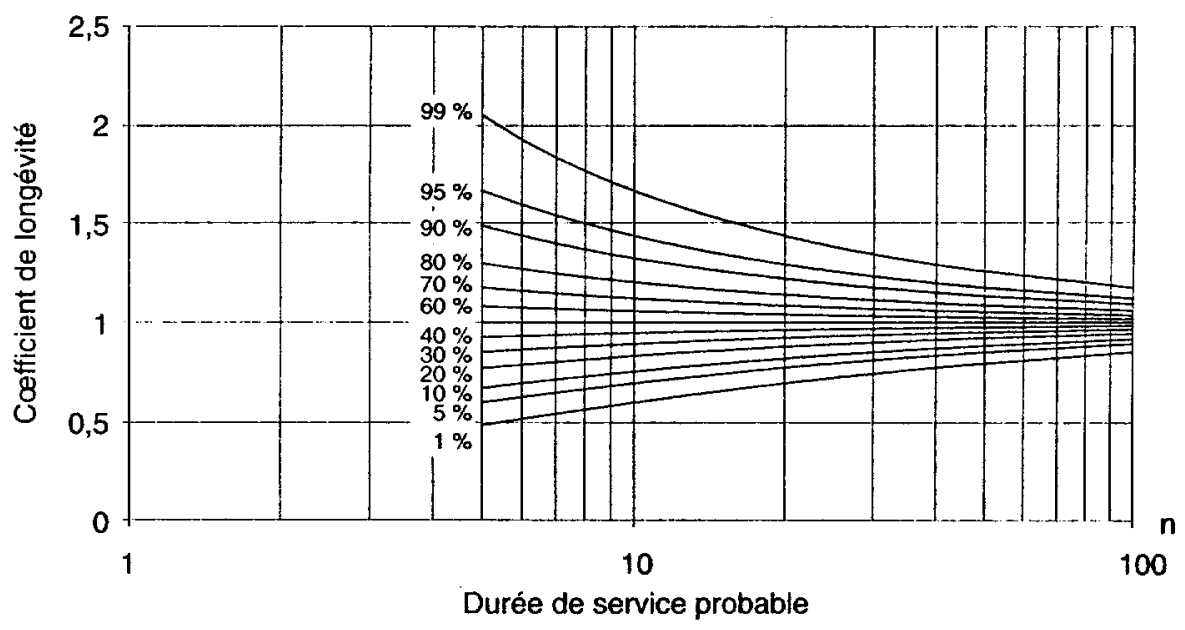

Figure 13 Abaque de prédiction des fluctuations des durées de service des lacs et barrages collinaires.

Prediction diagram relative to the fluctuation of the small and hilly dams service duration.

Tableau 5 Longévités probables pour $n=50$ ans.

Table 5 Probable time lengths for $n=50$ years.

\begin{tabular}{|lccccccccccccc|}
\hline Quantile & $1 \%$ & $5 \%$ & $10 \%$ & $20 \%$ & $30 \%$ & $40 \%$ & $50 \%$ & $60 \%$ & $70 \%$ & $80 \%$ & $90 \%$ & $95 \%$ & $99 \%$ \\
$\mathrm{U}_{\mathrm{F}}$ & $-2,33$ & $-1,65$ & $-1,28$ & $-0,84$ & $-0,52$ & $-0,25$ & 0 & 0,25 & 0,52 & 0,84 & 1,28 & 1,65 & 2,33 \\
$\mathrm{C}_{\mathrm{I}, \mathrm{F}}$ & 0,80 & 0,85 & 0,88 & 0,92 & 0,95 & 0,98 & 1 & 1,02 & 1,05 & 1,09 & 1,13 & 1,17 & 1,26 \\
Durée probable & 40 & 43 & 44 & 46 & 48 & 49 & 50 & 51 & 53 & 54 & 57 & 59 & 63 \\
\hline
\end{tabular}

\subsection{Analyse de sensibilité et validation régionale}

Nous avons fait varier, un à un, les paramètres de pluie qui ont servi au calage du générateur (valeur double ou réduite de moitié pour chaque paramètre). Ainsi nous avons simulé des pluies ayant des caractéristiques différentes par rapport à celles observées (plus ou moins abondantes, plus ou moins durables, plus ou moins intenses etc.) et nous avons analysé dans chaque cas les écarts engendrés au paramètre de calage régional $\alpha$ par rapport à la valeur initiale (tabl. 6). Bien que les hauteurs d'eau et l'érosivité annuelles ( $H$ et $R$ ) soient souvent très sensibles aux variations des paramètres du modèle (notamment à $H A$ ), le paramètre de calage $\alpha$ est par contre peu sensible et n'engendre pas d'écarts manifestes à la valeur initiale.

D'un autre côté, nous avons observé que le paramètre $E . T . \log \left(x-x_{0}\right)$ est à peu près constant pour les séries de débits solides mesurés aux trois bassins versants ; compris entre 0,33 et 0,36 environ, avec une moyenne de l'ordre de 0,34 . Cet aléa étant faible devant la diversité des cas étudiés (régions, sites, tailles et natures des bassins versants etc.), il est donc raisonnable de supposer, que le paramètre régional $\alpha=0,3$ aurait une faible variabilité spatiale, sachant que la valeur de $\alpha=0,3$ est liée aux valeurs «E.T. $\log \left(x-x_{0}\right)$ ». De fait, le " grossis- 
Tableau 6 Analyse de sensibilité du paramètre régional $\alpha$ aux variations des paramètres du générateur.

Table 6 Sensitivity analysis of the $\alpha$ regional parameter to the fluctuations of the parameters of the generator.

\begin{tabular}{|lccc|}
\hline Réduction de moilié & Hmm & R & \multicolumn{1}{c|}{$\alpha$} \\
\hline NP & $-50 \%$ & $-50 \%$ & $+8 \%$ \\
HA & $-46 \%$ & $-77 \%$ & $-12 \%$ \\
DA & $-5 \%$ & $-6 \%$ & $-20 \%$ \\
NA & $-19 \%$ & $-21 \%$ & $0 \%$ \\
RA & $-2 \%$ & $-23 \%$ & $+4 \%$ \\
\hline Doublement & Hmm & \multicolumn{1}{c}{ R } & $\alpha$ \\
\hline NP & $+100 \%$ & $+111 \%$ & $-16 \%$ \\
HA & $+98 \%$ & $+358 \%$ & $+12 \%$ \\
DA & $+30 \%$ & $+64 \%$ & $+16 \%$ \\
NA & $+96 \%$ & $+127 \%$ & $-24 \%$ \\
RA & $-2 \%$ & $+43 \%$ & $-16 \%$ \\
\hline
\end{tabular}

sement " de chacune des 3 séries de débits solides, à l'aide du même principe de génération stochastique (génération de 1000 ans), appliqué aux lois d'ajustement (fig. 5, 6 et 8), permet de trouver après calculs, $\alpha=0,33$ pour oued El Hissiane, $\alpha=0,38$ pour oued Mellègue, et $\alpha=0,40$ pour oued Zeroud, soit des valeurs assez voisines de celle retenue. Même si elles sont supérieures à la valeur initiale, les résultats ne sont que peu sensibles, et les écarts de longévité engendrés sont marginaux.

II semble donc qu'il y a une faible variabilité spatiale des fluctuations interannuelles de la dynamique temporelle de l'érosivité des pluies et de l'érosion conséquente (variabilité spatiale des moyennes mais pas des écarts). Ainsi ce qui ferait la différence d'un bassin versant à un autre, c'est l'activité érosive moyenne annuelle, qui dépendra des conditions de l'environnement naturel et humain du bassin (sol, pente, végétation, pratiques culturales...), et qui permettent de déterminer la durée de service moyenne probable. Les aléas par rapport à ces valeurs moyennes, sont liés surtout à l'agressivité climatique des pluies, notamment les plus extrêmes. Ces aléas semblent sensiblement homogènes et assez constants d'un point géographique à un autre. Du moins, on peut l'admettre pour une région aussi peu váste que celle où sont programmés et réalisés les ouvrages en question.

\section{5 - CONCLUSION}

Ne disposant pas pour le moment de suffisamment de séries d'observations statistiquement exploitables, pour analyser les conséquences de la dynamique climatique érosive sur les réservoirs collinaires, on génère des échantillons de pluie de tailles suffisamment grandes et on étudie leurs caractéristiques particulières d'érosivité. Le grossissement des observations par la multiplication des échantillons et l'agrandissement de leur taille, ne génère pas plus d'informations que celles disponibles, par contre il permet de contourner les problèmes pratiques de l'échantillonnage, et nous éclaire sur quelques problèmes particuliers relatifs à la dynamique érosive du climat. 
Cependant, outre les hypothèses émises que nous aurions souhaité conforter par de plus larges exemples, certains aspects de la démarche paraissent discutables. Par exemple, notre approche n'apporte pas de réponses pour les problèmes tout à fait réels dus aux conséquences des mécanismes et des facteurs de la dynamique érosive dans l'envasement des réservoirs. Sans pour autant les ignorer, on considère toutefois que leur importance et leur influence sont à approcher lors de l'estimation de rythme probable d'envasement, qui demeure une donnée essentielle avant de calculer des fourchettes quantiliques fiables.

En se focalisant sur les bilans statistiques à long terme, nous retiendrons donc, et dans la limite de la validation de nos résultats par l'observation, les enseignements suivants :

- Bien entendu, la durée de service d'un ouvrage ne peut être considérée comme une valeur exacte qu'on détermine par avance. Cette durée de service est une variable aléatoire qui dépend essentiellement de la chronologie de l'agressivité climatique (érosivité des pluies, événements extrêmes...) durant les années qui suivront la mise en service de l'ouvrage; elle s'apprécie donc en terme de probabilité.

- On remarque que plus la durée de service est courte, plus il y a des risques de se tromper (forte incertitude). Inversement, plus grand est le réservoir, plus grande est sa durée de service probable, et plus sûre est l'estimation de cette durée. Au-delà de 20 à 30 ans, la durée a tendance à homogénéiser les séries, et conduit à de faibles écarts de fluctuation. Dans ces cas, les risques de "mort subite " dues à un orage semblent s'éloigner. La fiabilité de la méthode augmente donc avec la taille et l'importance de l'ouvrage, c'est-à-dire avec l'importance des investissements.

- D'autre part, on remarque, que les distributions statistiques des cumuls annuels ont une dissymétrie positive, les chances d'augmentation de la longévité sont donc plus importantes que les chances de réduction de celle-ci. Ceci n'est pas incompatible avec les aléas climatiques et hydrologiques que nous connaissons. Les années sèches ou hydrologiquement déficitaires étant plus fréquentes que les années humides, elles engendrent de rares remplissages des réservoirs, qui ont par conséquent moins de chance d'accueillir des sédiments. Toutefois cette longévité, due aux faibles chances de remplissage, se ferait au détriment de la fonctionnalité et de l'utilité du réservoir s'il est trop fréquemment à sec.

- Enfin, il apparaît que variabilité spatiale et irrégularité temporelle agissent en opposition, et qu'à l'irrégularité temporelle extrême des séries de débit solide, s'oppose une faible variabilité spatiale de cet aléa, dont l'influence est ressentie en tout point géographique d'une manière constante.

Manifestement cette méthode, bien qu'assez approximative, devrait aider, bien plus que l'empirisme ou l'arbitraire, à l'aménagement et la gestion des réservoirs collinaires. Sa mise en application, sera sans doute l'occasion d'éclaircir et de valider a posteriori ses points d'appui...

\section{REMERCIEMENTS}

Ce travail a été réalisé notamment à partir des données collectées par l'équipe hydrologique de la Mission ORSTOM en Tunisie. Les auteurs expriment leur gratitude aux responsables respectifs de l'hydrologie, H. CAMUS pour son soutien, et J. ALBERGEL pour ses précieux commentaires. 


\section{RÉFÉRENCES BIBLIOGRAPHIQUES}

ALBERGEL, J., REJEB, N. (1997). (à paraître) Les lacs collinaires en Tunisie: Enjeux, contraintes et perspectives. Compte rendu à l'Académie d'Agriculture de France.

BOURGES, J., BOUZAIANE, S. (1973). Les crues exceptionnelles du $12 / 12 / 73$, sur loued Zita. Direction des Ressources en eau. Note interne.

BOUZAIANE, S., LAFFORGUE, A., CAMUS, $H_{\text {., }}$ BENZARTI, Z. (1986). Monographie hydrologique des oueds Zeroud et Merguellil. Direction Générale des Ressources en Eau.

ClAUDE, J., ChARTIER, R. (1977). Mesure de l'envasement dans les retenues de six barrages en Tunisie, campagne de 1975. Cahier ORSTOM série hydrologie. XIV(1), 3-35.

CRUETTE, J., RODIER, J.A. (1971). Mesure de débits de l'oued Zeroud pendant les crues exceptionnelles de l'automne 1969. Cahier ORSTOM série hydrologie. XIII(1), 33-64.

DELHOUME, J.P. (1985). Étude en milieu méditerranéen semi-aride. Ruissellement et érosion en zone de piedmont de Tunisie centrale (Djebel Semmama). Résultats 1976 à 1981. Ministère de l'agriculture. Direction des sols, $153 \mathrm{p}$.

GHORBEL, A. (1991). Guide pratique des calculs hydrologiques. Direction Générale des Ressources en Eau, 61p.

HEUSCH, B. (1970). L'érosion du pré-Rif. Une étude quantitative de l'érosion hydraulique dans les collines marneuses du pré-Rif occidental. Annales de la recherche forestière au Maroc, numéro spécial, Études sur l'érosion, (12), Rabat, 9-176.

LABORDE, J.P. (1984). Analyse des données et cartographie automatique en hydrologie : éléments d'hydrologie lorraine. Thèse Doctorat d'état. Institut National Polytechnique de Lorraine. ENSGAPMN, 484 p.

MASSON, J.M. (1971). L'érosion des sols par l'eau en climat méditerranéen. Méthodes expérimentales pour l'étude des quantités de terre érodée à l'échelle du champ. Thèse Docteur-Ingénieur. Université des Sciences et Techniques du Languedoc, CNRS $n^{\circ}$ AO 5445, 213 p.

MASSON, J.M. (1980). Mesure de l'agressivité des pluies en rapport avec l'érosion des sols. La météorologie $V^{\oplus}$ série $n^{\circ} 20$, 327-324.

MORGAN, R.P.C. (1986). Soil erosion and conservation. Ed. Longman Scientific and Technical, 298 p.

POUYAUD, B., ALBERGEL, J., CAMUS, H., CHEVALLIER, P., LAMACHERE, J.M. (1995). Réhabilitation des régimes hydriques et hydrologiques des terres semi-arides dégradées. In : L'homme peut-jl refaire ce qu'il a défait? John Libbey Eurotext. Paris, 49-64.

ROOSE, E. (1976). Les facteurs de l'érosion hydrique en Afrique Tropicale. Études sur petites parcelles expérimentales de sol. Revue de géographie physique et de géologie dynamique. XVIII(4), 365-374.

TALINEAU, J.C., SELMI, S., ALAYA, K. (1994). Lacs collinaires en Tunisie semiaride. Sécheresse, 5(4), 251-256.

TODOROVIC, P., YEVJEVICH, V. (1969). Stochastic process of precipitation. Hydrologie papers, 35 . Colorado States University, Fort Collins.

TOURASSE, P. (1981). Analyses spatiales et temporelles de précipitations et utilisation opérationnelle dans un système de prévision des crues. Thèse Docteur-Ingénieur. Institut National Polytechnique de Grenoble.

VOGT, H. (1991). Quelques problèmes concernant les méthodes de recherche en érosion des sols. Actes du colloque sur l'érosion des sols et l'envasement des barrages. ANRH, 10-15.

WISCHMEIER, W.H., SMITH, D.D. (1958) Évaluation of factors in the soil loss equation. Agr. Eng., 39, 458-462.

WISCHMEIER, W.H. (1959). A rainfall erosion index for a universal soil-loss équation. Soil Sc. Soc. Amer. Proc., 23, 246-249.

WISCHMEIER, W.H., SMITH, D.D. (1960). A universal soil-loss estimating équation to guide conservation farm planning. $7^{\text {th }}$ Intern. Congr. Soil Science, 1, 418-425.

ZAHAR, Y. (1994). Modélisation spatiale et temporelle des précipitations extrêmes et érosives en Tunisie centrale, en vue de l'aménagement des petits bassins hydrologiques. Thèse de Doctorat. Université de Nice-Sophia Antipolis, 416 p. 